\title{
Endothelial barrier dysfunction, loss of microcirculatory flow and organ failure in sepsis: current clinical view of sepsis and links to basic research
}

\section{Authors:}

Natalie Burkard

Michael Meir

Sven Flemming

Christoph-Thomas Germer

Nicolas Schlegel

\section{Affiliation:}

University of Wuerzburg, Department of general, visceral, vascular and paediatric surgery (Department of Surgery I), Oberduerrbacherstraße 6, D-97080 Wuerzburg, Germany

Sources of support: These studies were supported by a grant from the Deutsche Forschungsgemeinschaft SCHL 1962/2-1; SCHL 1962/4-1

Conflict of interest: None of the authors has a conflict of interest to declare

Running Head: Sepsis and endothelial barrier

\section{Corresponding author:}

Priv.-Doz. Dr. Nicolas Schlegel

Department of general, visceral, vascular and paediatric surgery (Department of Surgery I), Oberduerrbacherstraße 6, D-97080 Wuerzburg, Germany;

Schlegel_N@ukw.de

\section{Authors e.mails:}

Natalie Burkard: Burkard_N@ukw.de

Michael Meir: Meir_M@ukw.de

Sven Flemming: Flemming_S@ukw.de

Christoph-Thomas Germer:

Germer_C@ukw.de

\begin{abstract}
Sepsis and septic shock are major healthcare problems, affecting millions of people around the world each year, with an increasing incidence. In the last decades, clinical and basic research have significantly improved the understanding of the complex pathophysiology of sepsis. This led to the new sepsis definition ("Sepsis-3") which abandoned the view that sepsis is predominately defined by the various immunological responses. Rather, sepsis is now defined as life-threatening organ dysfunction caused by a dysregulated host response to infection. This view with a focus on the establishment of organ failure warrants the intensified research for therapeutic attempts to prevent and treat organ failure in sepsis. In this context, it is increasingly recognized that microvascular endothelial barrier dysfunction followed by loss of microcirculatory flow are key events leading to organ failure that should be therapeutically targeted. This review summarizes the current clinical view of the novel sepsis definition and connects it to basic research aspects of endothelial barrier regulation and loss of microcirculatory flow and thus organ protection.
\end{abstract}




\section{Introduction}

Sepsis is a clinical syndrome that is characterized by numerous infectiontriggered reactions of the whole body leading to a severe state of critical illness with a high mortality rate. Although the true incidence of sepsis is unknown, even conservative estimations indicate that sepsis is the leading cause of mortality and critical illness worldwide (1)(2). Therefore, severe sepsis and septic shock are major healthcare problems, affecting millions of people around the world each year with an increasing incidence (3).

During the last years significant progress has been made in the understanding of the complex immunological and pathophysiological processes underlying septic inflammation (4). However, most of the studies that aimed to translate basic research into clinical trials in order to influence immunological reactions in sepsis failed (4)(5). Given that sepsis mortality remained high there was a need for a novel and more differentiated view on sepsis pathophysiology. That has been considered recently by the third international consensus conference for Sepsis and Septic Shock ("Sepsis-3"). In brief, the definitions of this conference pointed out that in addition to infection, the dynamics in developing organ failure are critical for the definition or diagnosis of sepsis. It is increasingly recognized that the breakdown of microvascular endothelial barrier function followed by loss of microcirculatory flow are key events leading to organ failure. However, despite its importance, no clinically applicable approaches are yet available to address this basic problem of sepsis and critical illness. This review aims to summarize the novel sepsis definitions and discuss them in the context of endothelial barrier dysfunction and loss of microcirculatory flow.

\section{1) Current clinical view of sepsis}

\subsection{Sepsis definitions before 2016}

Sepsis was already known in antiquity which is reflected by the fact that the term "sepsis" derived from the Greek word for "rotting". The view on sepsis was further refined when it became obvious that infection is the cause for establishing a disease or syndrome called sepsis. However, it took time until 1992 when a consensus conference established for the first time clear definitions of sepsis and septic shock (6). These definitions were based on the view that sepsis resulted from a host's systemic inflammatory response syndrome (SIRS) to infection (Table 1).

Table 1 (modified according to (3))

\begin{tabular}{|l|}
\hline SIRS (Systemic Inflammatory Response Syndrome) \\
\hline Two or more of: \\
Temperature $>38^{\circ} \mathrm{C}$ or $<36^{\circ} \mathrm{C}$ \\
Heart rate $>90 / \mathrm{min}$ \\
Respiratory rate $>20 / \mathrm{min}$ or $\mathrm{PaCO}_{2}<32 \mathrm{mmHg}(4.3 \mathrm{kPa})$ \\
White blood cell count $>12000 / \mathrm{mm}^{3}$ or $<4000 / \mathrm{mm}^{3}$ or $>10 \%$ immature bands \\
\hline
\end{tabular}

Sepsis was diagnosed, if an infection occurs and two of four SIRS criteria (temperature $>38^{\circ} \mathrm{C}$ or $<36^{\circ} \mathrm{C}$, heart rate $>90 /$ min, respiratory rate $>20 /$ min or $\mathrm{PaCO}_{2}$ $<32 \mathrm{mmHg}$ or white blood cell count $>12.000 / \mathrm{mm}^{3}$ or $<4.000 / \mathrm{mm}^{3}$ or $>10 \%$ immature cells) could be determined. Severe sepsis was defined, when in addition to the SIRS criteria, organ dysfunction became evident. The progress from severe sepsis to septic shock was defined as "sepsis-induced hypotension persisting despite adequate fluid resuscitation". 
These definitions were revised in 2001 (7). The list of diagnostic criteria was extended but the definitions of sepsis, severe sepsis and septic shock remained mostly unchanged.

Due to the large number of new
findings on the pathophysiology,
management and epidemiology of sepsis
during the last years and due to the problem
that the SIRS criteria are very sensitive and not very specific, a task force consisting of 19 specialists of critical care medicine, infectious disease, surgery and pulmonology was founded in January 2014 by "The European Society of Intensive Care Medicine" and "The Society of Critical Care Medicine". This resulted in new consensus definitions of Sepsis called "Sepsis-3" that were published in 2016 (3). The key points of "Sepsis-3" are shown in Table 2.

Table 2: Key concepts of sepsis (modified according to (3))

\section{Key concepts of sepsis}

Sepsis is the primary cause of death from infection, especially if not recognized and treated promptly. Its recognition mandates urgent attenuation

Sepsis is a syndrome shaped by pathogen factors and host factors (e.g. sex, race, and other genetic determinants, age, comorbidities, environment) with characteristics that evolve over time. What differentiates sepsis from infection is an aberrant or dysregulated host response and the presence of organ dysfunction

Sepsis-induced organ dysfunction may be occult: therefore, its presence should be considered in any patient presenting with infection. Conversely, unrecognized infection may be the cause of new-onset organ dysfunction. Any unexplained organ dysfunction should thus raise the possibility of underlying infection

The clinical and biological phenotype of sepsis can be modified by preexisting acute illness, long-standing comorbidities, medication, and interventions

Specific infections may result in local organ dysfunction without generating a dysregulated systemic host response

\subsection{Current sepsis definition (Sepsis-3)}

Sepsis is now defined as "lifethreatening organ dysfunction caused by a dysregulated host response to infection" (Table 3) (8).

Table 3: New terms and definitions (modified according to (3))

\section{New Terms and Definitions}

Sepsis is defined as life-threatening organ dysfunction caused by dysregulated host response to infection Organ dysfunction can be identified as an acute change in total SOFA score $\geq 2$ points consequent to the infection

In lay terms, sepsis is a life-threatening condition that arises when the body`s response to an infection injures its own tissue and organs

Patients with suspected infection who are likely to have prolonged ICU stay or to die in the hospital can be promptly identified at the bedside with qSOFA (quick SOFA)

Septic shock is a subset of sepsis in which underlying circulatory and cellular/metabolic abnormalities are profound enough to substantially increase mortality

Patients with septic shock can be identified with a clinical construct of sepsis with persisting hypotension requiring vasopressors to maintain MAP $\geq 65 \mathrm{mmHg}$ and having a serum lactate level $\geq 2 \mathrm{mmol} / /(18 \mathrm{mg} / \mathrm{dl})$ despite adequate volume resuscitation. With these criteria, hospital mortality is in excess of $40 \%$

The new definition emphasizes the development of organ dysfunction, and indicates that the underlying patho- mechanism is more complex than assumed. The initial understanding of sepsis as infection with at least 2 of 4 SIRS criteria 
focused solely on the excess of inflammation. As mentioned above, it turned out that these SIRS criteria are very sensitive but not specific. Improved understanding of sepsis pathobiology pointed out, that sepsis is a multifaceted host response to a pathogen that may be significantly amplified by endogenous factors (9)(10). Therefore, the most important change in the third international consensus definitions for sepsis and septic shock (Sepsis-3) is that the SIRS criteria are no longer crucial for the diagnosis of sepsis since SIRS criteria are present in many hospitalized patients, including those who never develop infection and never evolve adverse outcomes (poor discriminant validity) (11). Furthermore, several patients with infection and new organ failure do not achieve two of four SIRS criteria and develop any adverse effects with significant morbidity and mortality (poor concurrent validity) (12).

Important for the prognosis and diagnosis of sepsis is the severity of damages that cause organ dysfunction. This is reflected by the SOFA-score ("Sequential (Sepsis-Related) Organ Failure Assessment
Score") where organ dysfunction is determined by six parameters: breathing, coagulation, liver function, cardiovascular function, central nervous system (Glasgow Coma-Scale), and renal function. The task force recommends using a change in baseline of the SOFA score of two points or more to represent organ dysfunction. The combination of a change in SOFA score with a known or suspected infection leads to the diagnosis of sepsis. The baseline SOFA score should be zero. Patients with a SOFA score of two or more had an overall mortality risk of approximately $10 \%$ (8). A SOFA score of two or greater identified a 2to 2.5-fold increase risk of dying compared with patients with a SOFA score less than two (8).

For an easier bedside assessment, the quick SOFA ("qSOFA") score was established that should help to identify easily patients with suspected infections who are likely to have poor outcomes. The qSOFA criteria are altered mentation, systolic blood pressure $<100 \mathrm{mmHg}$, and respiratory rate $>22 /$ min (Table 4 ).

Table 4: Quick SOFA Criteria (modified according to (3))

\begin{tabular}{|l|}
\hline qSOFA (Quick SOFA) Criteria \\
\hline Altered mentation \\
\hline Systolic blood pressure $<100 \mathrm{mmHg}$ \\
\hline Respiratory rate $\geq 22 / \mathrm{min}$ \\
\hline
\end{tabular}

Finally, septic shock is now defined as "a subset of sepsis in which underlying circulatory and cellular metabolism abnormalities are profound enough to substantially increase mortality" (8). Patients with septic shock can be identified clinically by the requirement of vasopressor therapy to maintain mean arterial pressure above 65 $\mathrm{mmHg}$ and serum lactate levels above 2 $\mathrm{mmol} / \mathrm{l}$ despite adequate fluid management. The inclusion of lactate again emphasizes the cellular dysfunction which is primarily represented as an insufficient oxygen supply.

\section{2) Sepsis, organ failure and links to basic research}

2.1. Sepsis and general aspects leading to organ dysfunction

According to the above summarized Sepsis-3 definitions, it can be concluded that although sepsis has various manifestations, 
there is one major hallmark being pivotal for the diagnosis and prognosis: development of organ dysfunction. Therefore, it is evident that therapeutic strategies and research on pathophysiological alterations should further focus on mechanisms that induce organ failure.

In general, organ dysfunction is caused by low oxygen content within the cells. Oxygen content depends on ventilation, cardiovascular function and intact microcirculation. Microcirculatory dysfunction is one major cause for organ dysfunction and an independent prognosis factor (13). Under normal conditions, there is a dense network of capillaries. However, sepsis is associated with a decrease in capillary density in association with an increase in heterogeneity of perfusion (13). The decreased capillary density results in an increased diffusion distance for oxygen (14). Microvascular alterations can lead to cellular injury and impaired tissue oxygenation (15) resulting in metabolic dysbalance, organ dysfunction and death. The key event to induce loss of microcirculatory flow is the breakdown of endothelial barrier integrity which causes extravasation of fluid and changes of rheology. Due to the consecutive severe tissue edema, diffusion distance for oxygen is increased and, vice versa, postcapillary venules get mechanically compressed by the edema which further impairs microcirculatory flow. In conclusion, endothelial barrier leakage and microcirculatory dysfunction can be considered as a major cause for organ dysfunction and death in septic patients $(16)(17)(18)(19)$. So far, there is still no therapy to improve endothelial barrier dysfunction in sepsis although novel approaches are urgently needed. In the following, several aspects on cell biological and translational attempts will be discussed and how to address this problem in the clinical setting.

\subsection{Organization and regulation of endothelial barrier: What happens in inflammation?}

Septic inflammation is caused by microbial pathogens leading to a variety of cytokine responses accompanied with endothelial activation and dysfunction. Endothelial dysfunction is characterized by barrier disruption with increased extravasation of fluid followed by organ dysfunction, metabolic dysbalance and death (15)(16)(20)(21).

Under normal conditions, the endothelial barrier consists of cell-cell junctions and various noncellular components (e.g. the glycocalyx and extracellular matrix). The intercellular cleft is sealed by tight junctions (TJ) and adherens junctions (AJ) that are both also functionally coupled to cell-matrix contacts. TJs are required for the sealing of the intercellular cleft and are mainly composed of occludin and claudins such as claudin 5 which is the only claudin expressed in peripheral endothelium (22). The cytoplasmic domain of TJ proteins is associated with zonula occludens protein-1 (ZO-1) which links TJs to $\alpha$-catenin, spectrin and the actin cytoskeleton. Importantly, the mechanical strength and stability of the endothelial barrier is provided by AJs (23). The predominant role in this context plays the endothelium-specific adhesion molecule vascular-endothelial (VE-) cadherin. VEcadherin undergoes homophilic binding by transinteraction of its extracellular domain in a $\mathrm{Ca}^{2+}$-dependent manner. The cytoplasmatic domain of VE-cadherin has binding sites for p120 catenin as well as for $\beta$ - and $\gamma$-Catenin which tether the cadherin-catenin-complex to the actin cytoskeleton via $\alpha$-Catenin or other proteins (Figure 1). 


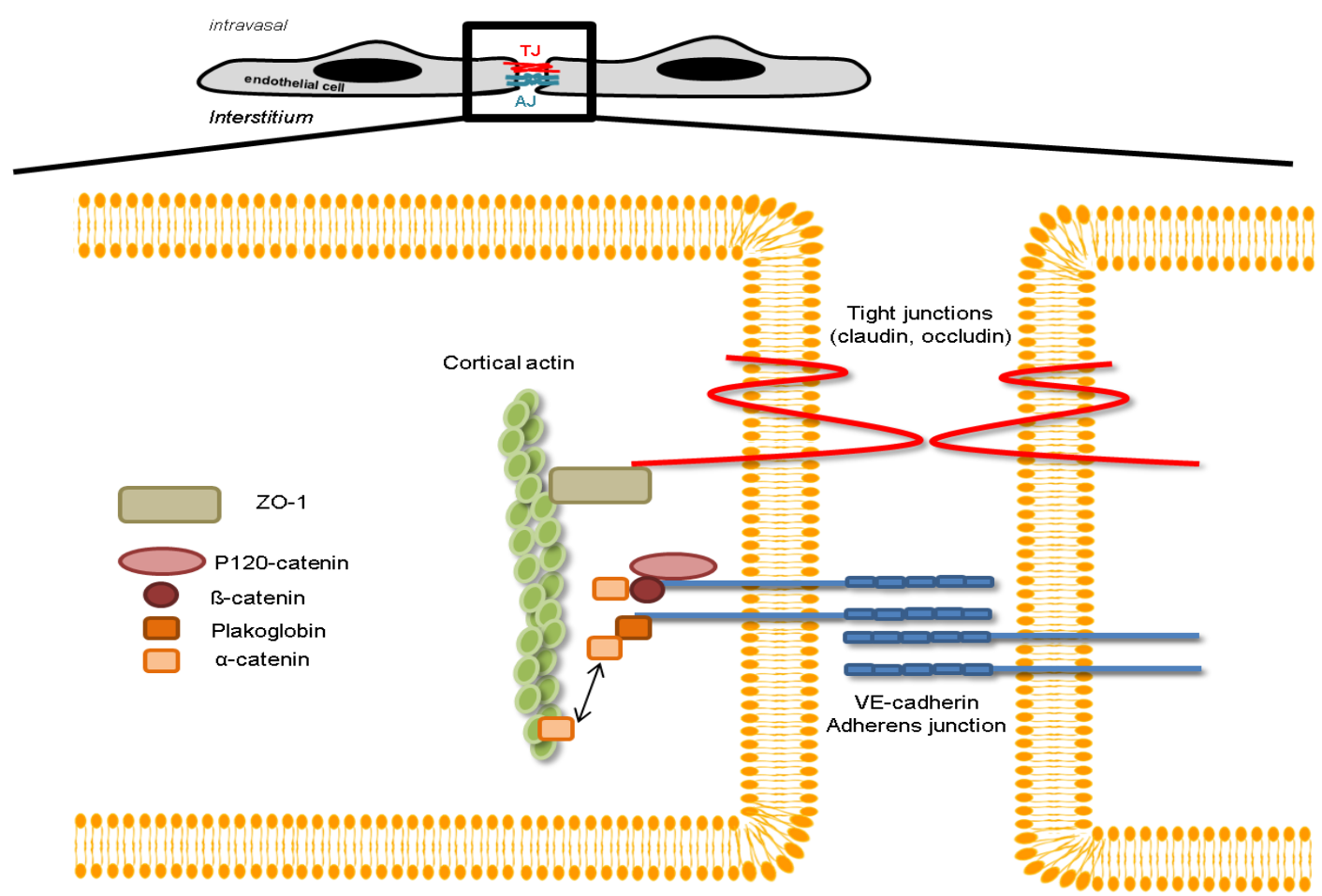

Figure 1: Endothelial barrier under normal conditions.

The endothelial barrier consists of endothelial cells that line all blood vessels. The intercellular cleft between endothelial cells is sealed by tight junctions (TJ) and adherens junctions (AJ). TJs are required for the sealing of the intercellular cleft while AJs that predominately consist of VE-cadherin provide the mechanical strength of the endothelial barrier. Both, TJ and AJ are connected to the cortical actin cytoskeleton via adaptor proteins.

The association of TJs and AJs with the actin cytoskeleton explains why intracellular signaling that affects actin dynamics is critically involved in the regulation of the endothelial barrier (24). Since the mechanical stability of the endothelial barrier is the prerequisite for its integrity, it is important to look at alterations of VE-cadherin-mediated adhesion/integrity in the context of inflammation. It can be assumed that the stabilization of VEcadherin-mediated adhesion/integrity is one of the key targets to therapeutically stabilize endothelial barrier function. Therefore, in the following we focus on the outstanding role of VE-cadherin in the regulation of the endothelial barrier function in inflammation (25).

\subsubsection{Binding partners/phosphoryla-} tion of VE-cadherin

The intracellular juxtamembrane and carboxyl-terminal domains of VE-cadherin interact directly with $ß$-catenin proteins, p120-catenin, and plakoglobin. Plakoglobin and $\beta$-catenin can bind $\alpha$-catenin, which regulates the actin cytoskeleton (25)(26)(27) and thereby modulate cell shape and motility (28). The cadherin-catenin complex is certainly required for full cellular control of endothelial permeability and junctional stabilization, although the precise molecular mechanism is still not known (25)(29). Additionally, the VE-cadherin-catenin complex plays an important role in the transduction of mechanical forces that shape endothelial cells exposed to blood pressure and shear stress. VE-cadherin is able to form 
a mechanosensor complex through binding to the junctional protein PECAM and VEGFR2 (30)(31). Src and other intracellular signals are activated through this complex and ensure the vascular homeostasis to maintain the correct endothelial attachment to the matrix and regulate blood flow.

Some preclinical trials with $\mathrm{Src}$ inhibitors in mice abolished the Vascular endothelial growth factor (VEGF)-induced increase in vascular permeability (32). However, since Src modulates several important cell functions (e.g. proliferation, survival, motility, and invasion), usage in clinical trials is not feasible because of its side-effects. Early clinical trials exist using an anti-VEGF receptor 2 antibody, which was shown to block VEGF-2-mediated breakdown of the endothelial barrier (33)(34). A pilot study to test the anti-VEGF antibody Bevacizumab in patients with septic shock was announced in 2010 (clinicaltrials.gov identifier NTCT01063010). The VEGF-antibody blocks VEGF-2 mediated breakdown of VEcadherin in endothelial cells. The trial was withdrawn in 2015 due to unknown reasons.

Phosphorylation of VE-cadherin plays also an important role in regulation of endothelial permeability (Figure 2).

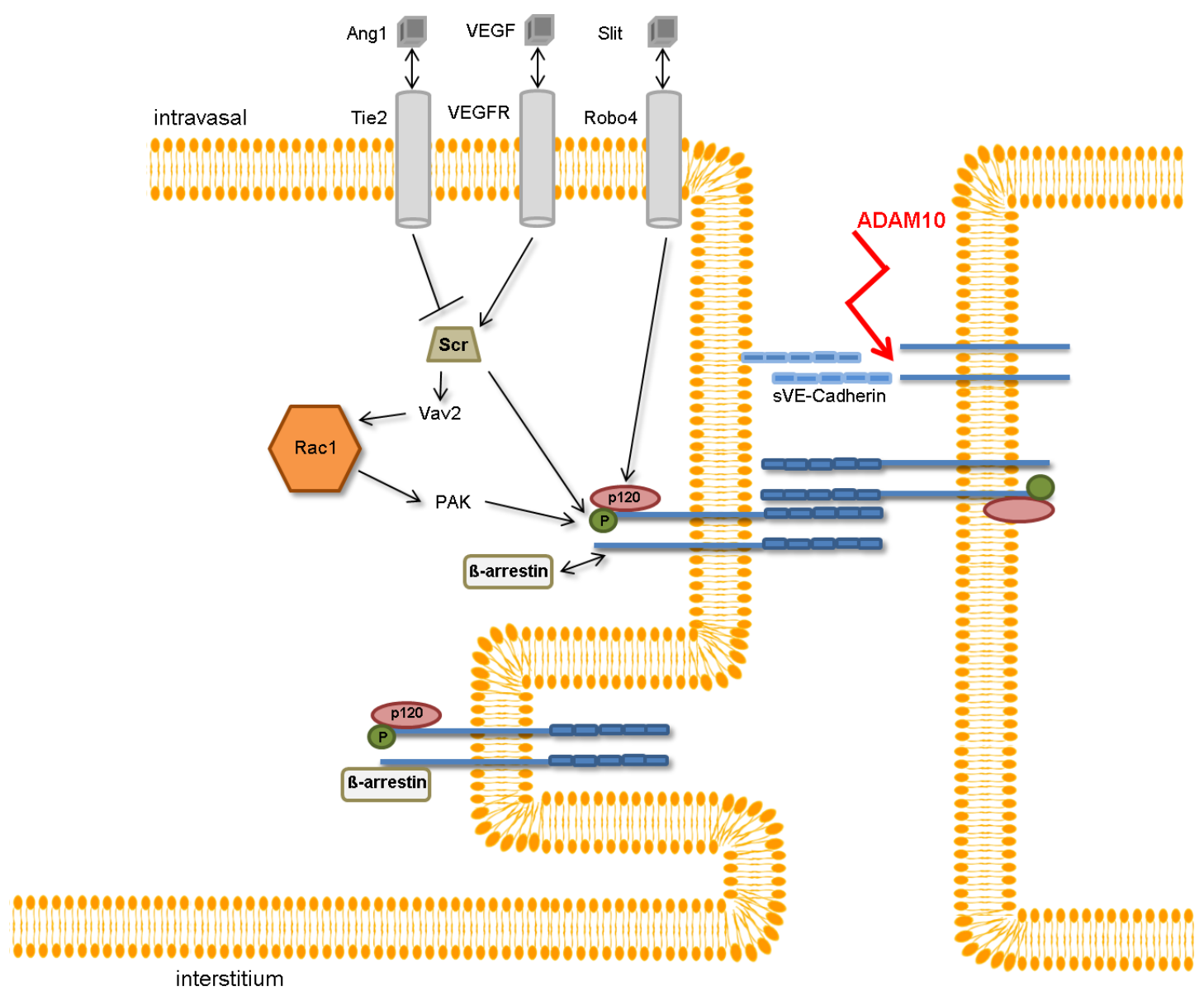

Figure 2: Phosphorylation, internalization and cleavage of VE-cadherin.

A signaling cascade (induced by VEGF) involving Scr kinase Vav2, and Rac1 leads to phosphorylation/internalization of VE-cadherin. VE-cadherin is phosphorylated either directly via Scr or indirectly via downstream signaling via Vav2. Phosphorylation of VE-cadherin leads to recruitment of $\beta$ arrestin and internalization of VE-cadherin through clathrin-coated vesicles. 
Internalization of VE-cadherin is inhibited via Slit/Robo4 pathway (by increased association of p120catenin) or via Ang1/Tie2 pathway (by sequestering Src and blocking its phosphorylation in response to VEGF).

ADAM10 cleaves VE-cadherin into two fragments and sVE-cadherin fragments block VE-cadherin binding and adhesion.

Tyrosine phosphorylation regulates the integrity of adherens junction protein VEcadherin (25). The binding of p120-catenin to VE-cadherin masks a dileucine motif that normally acts as a signal for clathrinmediated endocytosis of VE-cadherin. This prevents the translocation of VE-cadherin from the cell surface, where it is required to maintain normal cell-cell adhesion (26)(27). In this context, it is important to note which site is phosphorylated, since for example serine phosphorylation of VE-cadherin leads to recruitment of $\beta$-arrestin2 and its internalization through clathrin-coated vesicles (33) (Figure 2). This phosphorylation can be induced by several agents (e.g. by VEGF) which initiate a signaling cascade involving Src kinase, Vav2, and Rac. Additionally, the extent of phosphorylation of VE-cadherin might also be increased by inhibition of adherens junctions-associated phosphatases. In this context, the vascular endothelial protein tyrosine phosphatase (VE-PTP) appears to play a key role: The dissociation of VE-PTP from VE-cadherin is triggered by endotoxin VEGF, and the binding of leukocytes to endothelial cells in vitro and in vivo, suggesting that this process is of major importance for the destabilization of endothelial cell contacts under inflammatory conditions (35)(36).

\subsubsection{Internalization and cleavage}

Internalization of VE-cadherin is not only induced by phosphorylation, but also by Slit2N/Robo4 signaling. Slit is a family of secreted extracellular matrix proteins which can bind to the Robo4-receptor. Slit $2 \mathrm{~N}$ is a recombinant Slit fragment which stabilizes VE-cadherin at junctions by increasing p120-catenin binding and signaling through
Robo4 which inhibits VEGF-induced microvascular leakage by blocking Src activation (37)(16).

Furthermore, our group demonstrated that inflammation-induced endothelial barrier disruption results in an increase of soluble VE-cadherin fragments (sVEcadherin) released from endothelial cells (=cleavage of VE-cadherin) (38) (Figure 2). We and others identified ADAM10 (a disintegrin and metalloproteinase) being responsible for cleavage of VE-cadherin into two fragments, and showed that the release of the extracellular domains of VE-cadherin blocks VE-cadherin binding and adhesion (38)(39). Clinical data demonstrate that increased levels of sVE-cadherin in the blood of septic patients are associated with a negative outcome of these patients (40). As a clinical relevance of these findings, sVEcadherin may be used as a prognosis marker for sepsis or onset of microvascular barrier breakdown, respectively. However, this remains to be investigated in more detail.

\subsubsection{Cytoskeletal dynamics play a} critical role for VE-cadherin-dependent endothelial barrier stability

Apart from phosphorylation and internalization, actin dynamics are crucial for the regulation of endothelial permeability. Actin filaments are critical components of the cytoskeleton and can be found in several distinct structures. The cortical actin is a girdle of actin bundles which spans the entire circumference of endothelial cells and is thought to be associated with adherens and tight junctions via various adapter proteins (11). VEcadherin is linked to the actin cytoskeleton 
via associated catenins (41). Especially the cortical actin is dynamically regulated by small GTPases of the Rho family from which Rac1, Cdc42 and RhoA are the best characterized family members.

When the endothelial barrier is stabilized, cortactin is abundant at the cell border in aRac1-dependent manner (42)(43)(44)(45). Cortactin is an ubiquitously expressed tyrosine kinase target and has been implicated in cortical actin assembly and reorganization (46). Attenuated responses to barrier-protective stimuli were observed after cortactin knockdown, showing its functional relevance for endothelial permeability (47)(48)(49). Rac1 is thought to stabilize the cortical actin via LIM kinase (50). Another player to mention in this context is IQGAP1 (Ras GTPase-activating-like protein). IQGAP1, also known as p195, is an ubiquitously expressed protein that in humans is encoded by the IQGAPI gene. IQGAP1 is a scaffold protein involved in regulating various cellular processes ranging from organization of the actin cytoskeleton, transcription, and cellular adhesion to regulating the cell cycle. In endothelial cells, it has been proposed that Rac1 and $\mathrm{Cdc} 42$ regulate cadherin-mediated cell adhesion by sequestering IQGAP1 (51). Similary, regulation of VE-cadherin-mediated adhesion by activation of Rap1 which is a small GTPase of the Ras superfamily can in part be explained by IQGAP1 binding (52). IQAP1 inhibits $\alpha$-catenin binding to $\beta$ catenin resulting in impaired $\mathrm{F}$-actin linkage to VE-cadherin.

$\underline{2.2 .4 \text { Rac1 represents a central key }}$ player in the regulation of vascular permeability by various signaling events

As already mentioned above, different members of the Rho GTPase family (RhoA, Rac1, and Cdc42) (53)(54)(55) are key players in the regulation of vascular permeability. It was demonstrated that VE- cadherin clustering increases the concentration of Tiam1 (a guanine nucleotide exchange factor (GEF) for Rac1), and PAK (p21-activated kinase). PAK enhances Rac1 activity (25). Additionally, p120-catenin binding to VE-cadherin activates Vav2 (another specific GEF for Rac1). Finally, Rac1 activates different downstream targets, such as PAK and p190Rho-GTPase activating protein (p190RhoGAP), which in turn inhibits RhoA (56). Therefore, it can be assumed that Rac1 plays a key role for the maintenance of endothelial barrier function (55). In this context it is important to note that virtually all signaling pathways compromised in inflammation converge on alterations of Rac1 activity which leads to an increased microvascular permeability in vitro and in vivo (57).

According to this, therapeutic targeting of Rac1 would be the most promising approach to overcome endothelial barrier breakdown in septic inflammation. However, this is limited by the fact that direct Rac1 activators are absent to date. Therefore, it appears attractive to look on mediators or targets that indirectly influence Rac1 in order to stabilize the endothelial barrier.

In this context, several recent reports on the role of abl family tyrosine kinases in the regulation of Rac1 and endothelial permeability are interesting. The pharmacological inhibitor Imatinib is a small molecule which blocks the ATP-ase activity of the kinases c-Abl, Abl-related gene (Arg/Abl2), platelet-derived growth factor receptor (PDGFR), c-Kit, and discoid domain receptor-1 (58). Some clinical trials showed an association of Imatinib treatment with resolution of edema (59)(60)(61). Preclinical trials demonstrated that Imatinib reduces edema via preservation of endothelial barrier integrity (62) (63). Inhibition of Abl kinase activity causes an activation of Rac1 and Rap1 and thereby a stabilization of endothelial barrier, as 
mentioned before. Although Imatinib seems to be a good possibility for treatment of edema, it is associated with several side effects (like cardiac failure (64), subcutaneous edema after long-term treatment (65)).
Another mediator that regulates Rac1 activity is Sphingosin-1-Phosphate (S1P) which is secreted by thrombocytes and erythrocytes and acts via specific receptors (S1P receptor 1 to $\mathrm{S} 1 \mathrm{P}$ receptor 5) (Figure $3)$.

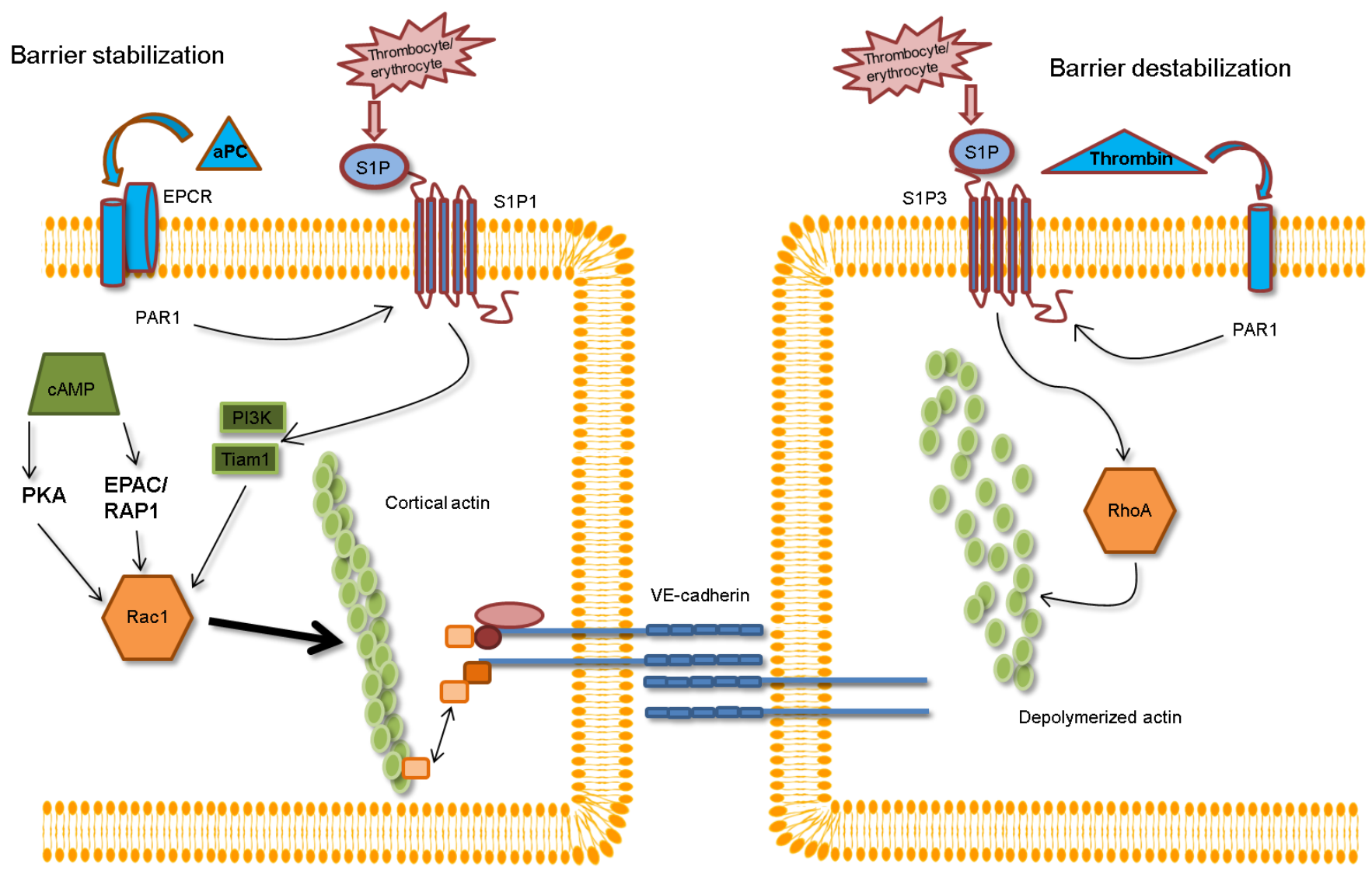

Figure 3: Rac1 and its role in the regulation of microvascular permeability

Barrier stabilization: aPC binds to its receptor which leads to activation of S1P1 receptor, PI3K/Tiam activation and finally to activation of Rac1. Additionally, S1P signaling results in VE-cadherin recruitment to the endothelial cell borders.

Barrier destabilization: Thrombin leads to activation of S1P3 receptor and via RhoA signaling to barrier disruption.

The cAMP signaling pathway also modulates Rac1 activity. Increased cAMP levels lead to PKA- and EPAC/Rap1-dependent activation of Rac1.

$\mathrm{S} 1 \mathrm{P}$ is produced through the phosphorylation of sphingosine by sphingosine kinases and enhances cortical actin formation in endothelial cells and thereby increases barrier integrity via several downstream targets (Rac1, cortactin, FAK, paxillin, actinin 1 and $4(66)(67)(68)(69)$ (22). In addition to its effects on actin, S1P signaling causes VE-cadherin recruitment to the endothelial cell junction resulting in increased integrity of the endothelial monolayer and decreased protein leakage 
(70). Furthermore, S1P protects the endothelial glycocalyx by inhibiting syndecan-1 shedding (68). Although the protective effects of S1P are mediated through the S1P1 receptor, thrombinmediated leak triggers the S1P3 receptor, leading to barrier disruption, in part through activation of RhoA [73][74] (Figure 3). The divergent effects of S1P may be an effect of dose or of location. Some preclinical trials showed a barrier protective effect of APC (activated protein C). APC leads to an increased S1P production via EPCR and PAR1 activation (73)(74). A clinical trial showed that the S1P carrier protein ApoM is reduced in septic patients (75)(76). Recently, Winkler and coworkers found a significant loss of S1P levels in close correlation to the disease severity of septic patients which indicates a causal relationship between decreased S1P levels and loss of microvascular barrier function in sepsis (77). Therefore, S1P is an important regulator of endothelial barrier function and further research is needed to clarify its mechanism of action and potential clinical utility.

An additional promising way to indirectly modulate Rac1 activity in inflammation is targeting the cAMP signaling pathway. Several studies showed that increased endothelial cAMP levels reduce both, permeability under resting conditions and increased permeability in response to inflammatory mediators (78). The ambivalent properties of cAMP are dependent on its generation in specific cellular compartments. In endothelial cells, cAMP is mainly synthesized by adenylatcyclase 6 . The adenylatcyclase 6 is activated by several receptors (adenosine 2-, prostaglandine E1-receptor or $B 2$ adrenergic receptor) and is localized at the plasmamembrane. cAMP is hydrolyzed by phosphodiesterase 3 or 4 , while phosphodiesterase 4 is the most abundant in human endothelium (79)(80). Our own studies and work by other researchers demonstrated that increased cAMP levels lead to PKA- and EPAC-dependent activation of Rac1 and thereby exert barrier protective effects (Figure 3). Importantly, in vivo studies showed that systemic application of the phosphodiesterase-4inhibitor rolipram increased endothelial cAMP in microvessels and attenuated capillary leakage and breakdown of microcirculatory flow in LPS-induced systemic inflammation in rats and in a model of polymicrobial sepsis $(81)(82)$.

Finally, the Angiopoetin-1/-2/Tie2 pathway plays another dominant role in endothelial barrier maintenance. Angiopoetin-1 activates the endotheliumspecific receptor Tie-2 which causes an activation of the phosphatidylinositol 3 kinase (PI3-kinase), and (through several effector molecules, like IQGAP1) finally activates Rac1 (83). Under resting conditions, endothelial barrier stabilization is Ang1/Tie-2-dependent (84) and under inflammatory conditions Ang1 blocks LPSinduced endothelial barrier breakdown (83).

Angiopoetin-2 (Ang2) is considered as the antagonist of Angiopoetin-1(Ang1). Ang2 is secreted by endothelial cells and stored (together with von Willebrand factor) in the so called "Weibel-Palade"-bodies. Ang2 is released after inflammatory stimuli. Ang2 prevents the Ang1 induced autophosphorylation of the Tie-2 receptor and thereby leads to endothelial disruption (85). Patients with severe systemic inflammation or sepsis show reduced levels of Ang1 but increased levels of Ang2. Therefore, Ang2 was shown to be an independent prognostic marker for the severity of sepsis (84)(86).

\section{Conclusion}

The new sepsis guidelines point out that the manifestation of organ failure is a fundamental criterion for sepsis diagnosis and prognosis. The cause for septic organ 
Sepsis and endothelial barrier

dysfunction is loss of oxygen supply. This is predominately initiated by the leakage of microvascular vessels which in turn is the most important factor to induce loss of microcirculatory flow. Therefore, targeting microvascular leakage will be a major goal in the future therapy of sepsis and further research on the underlying mechanisms and therapeutic attempts to interrupt this vicious circle are warranted to make the long awaited breakthrough in sepsis therapy. 


\section{References}

1. Vincent J-L, Marshall JC, NamendysSilva SA, François B, Martin-Loeches I, Lipman J, et al. Assessment of the worldwide burden of critical illness: the intensive care over nations (ICON) audit. Lancet Respir Med. 2014 May [cited 2016 Sep 19];2(5):380-6.

2. Fleischmann C, Scherag A, Adhikari NKJ, Hartog CS, Tsaganos T, Schlattmann P, et al. Assessment of Global Incidence and Mortality of Hospital-treated Sepsis. Current Estimates and Limitations. Am J Respir Crit Care Med. 2016 Feb;193(3):259-72.

3. Singer M, Deutschman CS, Seymour CW, Shankar-Hari M, Annane D, Bauer M, et al. The Third International Consensus Definitions for Sepsis and Septic Shock (Sepsis3). Jama. 2016;315(8):801-10.

4. Deutschman CS, Tracey KJ. Sepsis: current dogma and new perspectives. Immunity. 2014 Apr 17 [cited 2016 Sep 19];40(4):463-75.

5. Hotchkiss RS, Opal S. Immunotherapy for Sepsis - A New Approach against an Ancient Foe. N Engl J Med. 2010 Jun 30;363(1):879.

6. Bone R, Balk R, Cerra F, Dellinger R, Fein A, Knaus W, et al. accplsccm consensus conference for Sepsis and Organ Failure and. Chest. 1992;101:1644-55.

7. Levy MM, Fink MP, Marshall JC, Abraham E, Angus D, Cook D, et al. 2001 SCCM/ESICM/ACCP/ATS/SIS International Sepsis Definitions Conference. Crit Care Med.
2003;31(0090-3493 (Print)):1250-6.

8. Dellinger RP, Levy MM, Rhodes A, Annane D, Opal SM, Sevransky JE, et al. Surviving Sepsis Campaign. Int Guidel Manag Sev Sepsis Septic Shock. 2013;41(2):580-637.

9. Churpek MM, Zadravecz FJ, Winslow C, Howell MD, Edelson DP. Incidence and prognostic value of the systemic inflammatory response syndrome and organ dysfunctions in ward patients. Am J Respir Crit Care Med. 2015;192(8):958-64.

10. Kaukonen K-M, Bailey M, Pilcher D, Cooper DJ, Bellomo R. Systemic inflammatory response syndrome criteria in defining severe sepsis. N Engl J Med. 2015;372(17):1629-38.

11. Angus DC (Pitt), van der Poll $\mathrm{T}$. Severe sepsis and septic shock. N Engl J Med. 2013;369(9):840-51.

12. Wiersinga WJ, Leopold SJ, Cranendonk DR, van der Poll T. Host innate immune responses to sepsis. Virulence. 2014;5(1):36-44.

13. Moerer O, Quintel M. [Sepsis in adult patients - definitions, epidemiology and economic aspects]. Internist (Berl). 2009;50(7):788,790-794,796798.

14. Cinel I, Dellinger RP. Advances in pathogenesis and management of sepsis. CurrOpinInfectDis. 2007;20(0951-7375 (Print)):345-52.

15. Kumar P, Shen Q, Pivetti CD, Lee ES, Wu MH, Yuan SY. Molecular mechanisms of endothelial hyperpermeability: implications in inflammation. Expert Rev Mol Med. 
2009 Jun 30;11:e19-e19.

16. Goldenberg NM, Steinberg BE, Slutsky AS, Lee WL. Broken Barriers: A New Take on Sepsis Pathogenesis. Sci Transl Med. 2011 Jun 22;3(88):88ps25 LP-88ps25.

17. Fisher J, Douglas JJ, Linder A, Boyd JH, Walley KR, Russell JA. Elevated Plasma Angiopoietin-2 Levels Are Associated With Fluid Overload, Organ Dysfunction, and Mortality in Human Septic Shock. Crit Care Med. $2016 \mathrm{Jul}$;

18. Fitzgerald JC, Basu RK, AkcanArikan A, Izquierdo LM, Pineres Olave BE, Hassinger AB, et al. Acute Kidney Injury in Pediatric Severe Sepsis: An Independent Risk Factor for Death and New Disability. Crit Care Med. 2016 Aug;

19. Fjell CD, Thair S, Hsu JL, Walley KR, Russell JA, Boyd J. Cytokines and signaling molecules predict clinical outcomes in sepsis. PLoS One. 2013;8(11):e79207.

20. Lee WL, Liles WC. Endothelial activation, dysfunction and permeability during severe infections. Curr Opin Hematol. 2011;18:191-6.

21. Bazzoni G. Endothelial Cell-to-Cell Junctions: Molecular Organization and Role in Vascular Homeostasis. Physiol Rev. 2004;84(3):869-901.

22. Komarova Y, Malik AB. Regulation of endothelial permeability via paracellular and transcellular transport pathways. Annu Rev Physiol. 2010;72:463-93.

23. Dejana E, Orsenigo F. Endothelial adherens junctions at a glance. J Cell Sci. 2013 Jun;126(Pt 12):2545-9.
24. Waschke J, Curry FE, Adamson RH, Drenckhahn D. Regulation of actin dynamics is critical for endothelial barrier functions. Am J Physiol Heart Circ Physiol. 2005;288(3):H1296305.

25. Dejana E, Orsenigo F, Lampugnani MG. The role of adherens junctions and VE-cadherin in the control of vascular permeability. 2008;

26. Potter MD, Barbero S, Cheresh DA. Tyrosine phosphorylation of VEcadherin prevents binding of p120and beta-catenin and maintains the cellular mesenchymal state. J Biol Chem. 2005 Sep;280(36):31906-12.

27. Xiao K, Allison DF, Kottke MD, Summers S, Sorescu GP, Faundez V, et al. Mechanisms of VE-cadherin processing and degradation in microvascular endothelial cells. J Biol Chem. 2003 May;278(21):19199-208.

28. Weis WI, Nelson WJ. Re-solving the cadherin-catenin-actin conundrum. J Biol Chem. 2006 Nov;281(47):35593-7.

29. Giannotta M, Trani M, Dejana E. VEcadherin and endothelial adherens junctions: active guardians of vascular integrity. Dev Cell. 2013 Sep;26(5):441-54.

30. Conway DE, Breckenridge MT, Hinde E, Gratton E, Chen CS, Schwartz MA. Fluid shear stress on endothelial cells modulates mechanical tension across VEcadherin and PECAM-1. Curr Biol. 2013 Jun;23(11):1024-30.

31. Tzima E, Irani-Tehrani M, Kiosses WB, Dejana E, Schultz DA, Engelhardt B, et al. A mechanosensory complex that 
mediates the endothelial cell response to fluid shear stress. Nature. 2005 Sep;437(7057):426-31.

32. Weis SM, Cheresh DA. Pathophysiological consequences of VEGF-induced vascular permeability. Nature. 2005 Sep;437(7058):497-504.

33. Gavard J, Gutkind JS. VEGF controls endothelial-cell permeability by promoting the beta-arrestin-dependent endocytosis of VE-cadherin. Nat Cell Biol. 2006 Nov;8(11):1223-34.

34. Gavrilovskaya IN, Gorbunova EE, Mackow NA, Mackow ER. Hantaviruses Direct Endothelial Cell Permeability by Sensitizing Cells to the Vascular Permeability Factor VEGF, while Angiopoietin 1 and Sphingosine 1-Phosphate Inhibit Hantavirus-Directed Permeability . J Virol. 2008 Jun 26;82(12):5797-806.

35. Broermann A, Winderlich M, Block H, Frye M, Rossaint J, Zarbock A, et al. Dissociation of VE-PTP from VEcadherin is required for leukocyte extravasation and for VEGF-induced vascular permeability in vivo. J Exp Med. 2011 Nov;208(12):2393-401.

36. Vockel M, Vestweber D. How T cells trigger the dissociation of the endothelial receptor phosphatase VEPTP from VE-cadherin. Blood. 2013 Oct;122(14):2512-22.

37. London NR, Zhu W, Bozza FA, Smith MCP, Greif DM, Sorensen LK, et al. Targeting Robo4-Dependent Slit Signaling to Survive the Cytokine Storm in Sepsis and Influenza. Sci Transl Med. 2010 Mar 17;2(23):23ra19 LP-23ra19.

38. Flemming S, Burkard N, Renschler M, Vielmuth F, Meir M, Schick MA, et al. Soluble VE-cadherin is involved in endothelial barrier breakdown in systemic inflammation and sepsis. Cardiovasc Res. 2015 Jul;107(1):3244.

39. Schulz B, Pruessmeyer J, Maretzky T, Ludwig A, Blobel CP, Saftig P, et al. ADAM10 Regulates Endothelial Permeability and T-Cell Transmigration by Proteolysis of Vascular Endothelial Cadherin. Circ Res. 2008 May 22;102(10):1192 LP1201.

40. Zhang R-Y, Liu Y-Y, Li L, Cui W, Zhao K-J, Huang W-C, et al. Increased levels of soluble vascular endothelial cadherin are associated with poor outcome in severe sepsis. $\mathbf{J}$ Int Med Res. 2010;38(4):1497-506.

41. Lampugnani MG, Corada M, Caveda L, Breviario F, Ayalon O, Geiger B, et al. The molecular organization of endothelial cell to cell junctions: differential association of plakoglobin, beta-catenin, and alphacatenin with vascular endothelial cadherin (VE-cadherin). J Cell Biol. 1995 Apr;129(1):203-17.

42. Arce FT, Whitlock JL, Birukova AA, Birukov KG, Arnsdorf MF, Lal R, et al. Regulation of the Micromechanical Properties of Pulmonary Endothelium by S1P and Thrombin: Role of Cortactin. Biophys J. 2008 Jul 15;95(2):886-94.

43. Baumer Y, Drenckhahn D, Waschke J. cAMP induced Rac 1-mediated cytoskeletal reorganization in microvascular endothelium. Histochem Cell Biol. 2008;129(6):765-78.

44. Schlegel N, Burger S, Golenhofen N, Walter U, Drenckhahn D, Waschke J. 
The role of VASP in regulation of cAMP- and Rac 1-mediated endothelial barrier stabilization. Am J Physiol Cell Physiol. 2008;294(1):C178-88.

45. Waschke J, Burger S, Curry FRE, Drenckhahn D, Adamson RH. Activation of Rac-1 and $\mathrm{Cdc} 42$ stabilizes the microvascular endothelial barrier. Histochem Cell Biol. 2006;125(4):397-406.

46. Weed SA, Parsons JT. Cortactin: coupling membrane dynamics to cortical actin assembly. Oncogene. 2001 Oct;20(44):6418-34.

47. Jacobson JR, Dudek SM, Singleton PA, Kolosova IA, Verin AD, Garcia JGN. Endothelial cell barrier enhancement by ATP is mediated by the small GTPase Rac and cortactin. Am J Physiol Lung Cell Mol Physiol. 2006 Aug;291(2):L289-95.

48. Dudek SM, Jacobson JR, Chiang ET, Birukov KG, Wang P, Zhan X, et al. Pulmonary endothelial cell barrier enhancement by sphingosine 1phosphate: roles for cortactin and myosin light chain kinase. J Biol Chem. 2004 Jun;279(23):24692-700.

49. Zhao J, Singleton PA, Brown ME, Dudek SM, Garcia JGN. Phosphotyrosine protein dynamics in cell membrane rafts of sphingosine-1phosphate-stimulated human endothelium: role in barrier enhancement. Cell Signal. 2009 Dec;21(12):1945-60.

50. Bernard O. Lim kinases, regulators of actin dynamics. Int $\mathrm{J}$ Biochem Cell Biol. 2007;39(6):1071-6.

51. Noritake J, Watanabe T, Sato K, Wang S, Kaibuchi K. IQGAP1: a key regulator of adhesion and migration. $\mathbf{J}$ Cell Sci. 2005 May;118(Pt 10):208592.

52. Jeong H-W, Li Z, Brown MD, Sacks DB. IQGAP1 binds Rap1 and modulates its activity. J Biol Chem. 2007 Jul;282(28):20752-62.

53. Beckers CML, van Hinsbergh VWM, van Nieuw Amerongen GP. Driving Rho GTPase activity in endothelial cells regulates barrier integrity. Thromb Haemost. 2010 Jan;103(1):40-55.

54. Goddard LM, Iruela-Arispe ML. Cellular and molecular regulation of vascular permeability. Thromb Haemost. 2013 Mar;109(3):407-15.

55. Spindler V, Schlegel N, Waschke J. Role of GTPases in control of microvascular permeability. Cardiovasc Res. 2010;87(2):243-53.

56. Abe K, Rossman KL, Liu B, Ritola KD, Chiang D, Campbell SL, et al. Vav2 is an activator of Cdc42, Rac1, and RhoA. J Biol Chem. 2000;275(14):10141-9.

57. Schlegel N, Waschke J. cAMP with other signaling cues converges on Rac1 to stabilize the endothelial barrier - a signaling pathway compromised in inflammation. Cell Tissue Res. 2014;355(3):587-96.

58. Waller CF. Imatinib mesylate. Recent results cancer Res Fortschritte der Krebsforsch Prog dans les Rech sur le cancer. 2010;184:3-20.

59. Carnevale-Schianca F, Gallo S, RotaScalabrini D, Sangiolo D, Fizzotti M, Caravelli D, et al. Complete resolution of life-threatening bleomycin-induced pneumonitis after treatment with imatinib mesylate in a 
patient with Hodgkin's lymphoma: hope for severe chemotherapyinduced toxicity? J Clin Oncol. 2011 Aug;29(24):e691-3.

60. Su EJ, Fredriksson L, Geyer M, Folestad E, Cale J, Andrae J, et al. Activation of PDGF-CC by tissue plasminogen activator impairs bloodbrain barrier integrity during ischemic stroke. Nat Med. 2008 Jul;14(7):7317.

61. Armulik A, Genove G, Mae M, Nisancioglu MH, Wallgard E, Niaudet $\mathrm{C}$, et al. Pericytes regulate the bloodbrain barrier. Nature. 2010 Nov;468(7323):557-61.

62. Aman J, van Bezu J, Damanafshan A, Huveneers S, Eringa EC, Vogel SM, et al. Effective treatment of edema and endothelial barrier dysfunction with imatinib. Circulation. 2012 Dec;126(23):2728-38.

63. Chislock EM, Pendergast AM. Abl family kinases regulate endothelial barrier function in vitro and in mice. PLoS One. 2013;8(12):e85231.

64. Kerkela R, Grazette L, Yacobi R, Iliescu C, Patten R, Beahm C, et al. Cardiotoxicity of the cancer therapeutic agent imatinib mesylate. Nat Med. 2006 Aug;12(8):908-16.

65. Druker BJ, Talpaz M, Resta DJ, Peng $\mathrm{B}$, Buchdunger $\mathrm{E}$, Ford JM, et al. Efficacy and safety of a specific inhibitor of the BCR-ABL tyrosine kinase in chronic myeloid leukemia. N Engl J Med. 2001 Apr;344(14):1031-7.

66. Mehta D, Malik AB. Signaling mechanisms regulating endothelial permeability. Physiol Rev. 2006 Jan;86(1):279-367.
67. Garcia JG, Liu F, Verin AD, Birukova A, Dechert MA, Gerthoffer WT, et al. Sphingosine 1-phosphate promotes endothelial cell barrier integrity by Edg-dependent cytoskeletal rearrangement. J Clin Invest. 2001 Sep;108(5):689-701.

68. Zeng Y, Adamson RH, Curry F-RE, Tarbell JM. Sphingosine-1-phosphate protects endothelial glycocalyx by inhibiting syndecan- 1 shedding. Am J Physiol Heart Circ Physiol. 2014;306(3):H363-72.

69. Sun X, Shikata Y, Wang L, Ohmori $\mathrm{K}$, Watanabe $\mathrm{N}$, Wada $\mathrm{J}$, et al. Enhanced interaction between focal adhesion and adherens junction proteins: involvement in sphingosine 1-phosphate-induced endothelial barrier enhancement. Microvasc Res. 2009 May;77(3):304-13.

70. Adamson RH, Sarai RK, Altangerel A, Thirkill TL, Clark JF, Curry F-RE. Sphingosine-1-phosphate modulation of basal permeability and acute inflammatory responses in rat venular microvessels. Cardiovasc Res. 2010 Nov;88(2):344-51.

71. Singleton PA, Dudek SM, Ma S-F, Garcia JGN. Transactivation of sphingosine 1-phosphate receptors is essential for vascular barrier regulation. Novel role for hyaluronan and CD44 receptor family. J Biol Chem. 2006 Nov;281(45):34381-93.

72. Singleton PA, Moreno-Vinasco L, Sammani S, Wanderling SL, Moss J, Garcia JGN. Attenuation of vascular permeability by methylnaltrexone: role of $\mathrm{mOP}-\mathrm{R}$ and $\mathrm{S} 1 \mathrm{P} 3$ transactivation. Am J Respir Cell Mol Biol. 2007 Aug;37(2):222-31.

73. Kerschen EJ, Fernandez JA, Cooley 
BC, Yang X V, Sood R, Mosnier LO, et al. Endotoxemia and sepsis mortality reduction by nonanticoagulant activated protein C. J Exp Med. 2007 Oct;204(10):2439-48.

74. Wang L, Dudek SM. Regulation of vascular permeability by sphingosine 1-phosphate. Microvasc Res. 2009 Jan;77(1):39-45.

75. Kumaraswamy SB, Linder A, Akesson P, Dahlback B. Decreased plasma concentrations of apolipoprotein $\mathrm{M}$ in sepsis and systemic inflammatory response syndromes. Crit Care. 2012;16(2):R60.

76. Christoffersen C, Nielsen LB. Apolipoprotein $\mathrm{M}$--a new biomarker in sepsis. Crit Care. 2012 May;16(3):126.

77. Winkler MS, Nierhaus A, Holzmann M, Mudersbach E, Bauer A, Robbe L, et al. Decreased serum concentrations of sphingosine-1-phosphate in sepsis. Crit Care. 2015 Oct;19:372.

78. Michel CC, Curry FE. Microvascular permeability. Physiol Rev. 1999 Jul;79(3):703-61.

79. Lugnier C. Cyclic nucleotide phosphodiesterase (PDE) superfamily: a new target for the development of specific therapeutic agents. Pharmacol Ther. 2006 Mar;109(3):366-98.

80. Netherton SJ, Jimmo SL, Palmer D, Tilley DG, Dunkerley HA, Raymond DR, et al. Altered phosphodiesterase 3-mediated cAMP hydrolysis contributes to a hypermotile phenotype in obese JCR:LA-cp rat aortic vascular smooth muscle cells: implications for diabetes-associated cardiovascular disease. Diabetes. 2002 Apr;51(4):1194-200.

81. Flemming S, Schlegel N, Wunder C, Meir M, Baar W, Wollborn J, et al. Phosphodiesterase 4 inhibition dose dependently stabilizes microvascular barrier functions and microcirculation in a rodent model of polymicrobial sepsis. Shock. 2014 Jun;41(6):53745.

82. Schick MA, Wunder C, Wollborn J, Roewer N, Waschke J, Germer C-T, et al. Phosphodiesterase-4 inhibition as a therapeutic approach to treat capillary leakage in systemic inflammation. J Physiol. 2012 Jun;590(11):2693-708.

83. Mammoto T, Parikh SM, Mammoto A, Gallagher D, Chan B, Mostoslavsky G, et al. Angiopoietin-1 requires p190 RhoGAP to protect against vascular leakage in vivo. J Biol Chem. 2007 Aug;282(33):23910-8.

84. David S, Kumpers P, van Slyke P, Parikh SM. Mending leaky blood vessels: the angiopoietin-Tie2 pathway in sepsis. J Pharmacol Exp Ther. 2013 Apr;345(1):2-6.

85. Moss A. The angiopoietin:Tie 2 interaction: a potential target for future therapies in human vascular disease. Cytokine Growth Factor Rev. 2013 Dec;24(6):579-92.

86. Luz Fiusa MM, Costa-Lima C, de Souza GR, Vigorito AC, Penteado Aranha FJ, Lorand-Metze I, et al. A high angiopoietin-2/angiopoietin-1 ratio is associated with a high risk of septic shock in patients with febrile neutropenia. Crit Care. 2013;17(4):R169. 